Revista

\title{
Multi-Ensayos
}

Vol. 6, núm. 12

ISSN: 2412-3285

https://multiensayos.unan.edu.ni

DOI: https://doi.org/10.5377/multiensayos.v6i12.10116

\section{Nuevas perspectivas del lenguaje en la enseñanza de idiomas}

\section{New perspectives of language in language teaching}

Wilmer Lagos ${ }^{1}$

Recibido: 26 de junio de 2020. Aceptado: 24 de julio de 2020

\section{RESUMEN}

El presente ensayo tiene como objetivo hacer una revisión bibliográfica de las nuevas realidades de la pedagogía de idiomas, independientemente del contexto. En los últimos años, el concepto de lenguaje ha variado para incluir realidades del uso del idioma socialmente. Algunos académicos argumentan que los idiomas deben ser vistos como una actividad más que como una formación de palabras, más que simplemente un sistema al que recurrimos, como una parte ingeniosa de la vida social e intercultural en cualquier sociedad. De igual manera, académicos enfatizan que comprender como se usan los idiomas funcionalmente implica pensar en ideologías lingüísticas y su rol en el proceso de aprendizaje/enseñanza de idiomas. En la pedagogía de idiomas, entender que el lenguaje es un sistema adaptivo complejo con una función fundamental social es vital ya que implica entender cuál es la función que dicho idioma jugaría en diferentes aspectos de la sociedad. Lo que implica, consecuentemente, pensar que competencias que deben ser desarrolladas, dentro del aula de clase.

Palabras clave: lenguaje; pedagogía de idiomas; ideología lingüística.

\section{ABSTRACT}

The objective of this essay is to carry out a bibliographic review of the new realities of language pedagogy, regardless of the context. In the last few years, the concept of language has changed to include realities of language use socially. Some academics argue that languages should be seen as an activity rather than as a word formation, rather than simply a system that we turn to, as an ingenious part of social and intercultural life in any society. Similarly, academics emphasize that understanding how languages are used functionally implies thinking about linguistic ideologies and their role in the language learning - teaching process. In language pedagogy, understanding that language is a complex adaptive system with a fundamental social function is vital since it involves understanding what function that language would play in different aspects of society. This implies, consequently, thinking about what competencies should be developed within the classroom.

Keywords: language; language pedagogy; linguistic ideology.

1 Licenciado en Ciencias de la Educación con Mención en Ingles y Master en Recursos Pedagógicos. Ha trabajado a diferentes niveles de educación - Fundación Fabretto y FAREM-Estelí. Correo electrónico: gerlagos04@yahoo.com.

(c) 2020 Revista Multi-Ensayos. 


\section{INTRODUCCIÓN}

Con las crecientes conexiones entre personas de diferentes orígenes, el lenguaje se interpreta cada vez menos como un sistema autónomo inmóvil. El lenguaje ya no es esa estructura discreta discutida por Saussure o una gramática mental libre de contexto como señaló Chomsky (Garcia y Wei, 2014). El límite entre los idiomas ahora es borroso, donde se dibujan muchas interpretaciones sobre lo que constituye una u otra variación de un idioma ${ }^{1}$. En el estudio actual del lenguaje, por lo tanto, nos encontramos con un problema de terminología, El término 'lenguaje' se ha usado tradicionalmente para referirse al conocimiento y uso de enunciados y de símbolos escritos identificados como sonidos o signos semióticos, por ejemplo. Llegar con una definición única de qué es el lenguaje, hoy en día, es desconcertante. El lenguaje combina elementos en todos los niveles (por ejemplo, sistemas de sonido, sílabas) que a su vez se combinan en permutaciones específicas que se ordenan de manera predecible y siguen ciertos patrones. Además, el lenguaje explota las estructuras jerárquicas en varios niveles, dividiendo el significado en diferentes capas de especificidad (Tallerman y Gibson, 2012).

Si tuviéramos que dar una definición simple de qué es el lenguaje para la persona común, tendríamos que decir "es la forma en que las personas hablan entre sí cuando comparten el mismo sistema lingüístico complejo de comunicación", uno por ejemplo llamado inglés o español. Sin embargo, el lenguaje va más allá de esa simple definición. Estamos adquiriendo más conocimiento sobre los estilos de comunicación que amplían el concepto de sistemas lingüísticos, lo que a su vez presenta más complejidad en su evolución y, por lo tanto, en su enseñanza y aprendizaje. La declaración antes mencionada da lugar a un dilema fundamental que los académicos están experimentando en este momento en el campo de las pedagogías de la enseñanza de idiomas. ¿Cómo identificamos el lenguaje, sus límites y cómo lo enseñamos?

Sin lugar a dudas, el lenguaje es un sistema adaptativo complejo con una función fundamentalmente social. El lenguaje tiene diferentes definiciones dependiendo de dónde se base, sociolingüistas, antropología, evolución del lenguaje, psicolingüística, proceso del lenguaje, entre otros (Ellis y LarsenFreeman, 2009). Sin embargo, el lenguaje es posiblemente el primer símbolo utilizado para la interacción social. Por lo tanto, en este ensayo, el lenguaje será visto como un símbolo o recurso cuyos orígenes y aptitudes dependen de su papel en la vida social. El uso de este recurso (por ejemplo, signos, discurso) es individual; es decir, depende de las habilidades cognitivas, la conciencia, la historia, la interacción social y los antecedentes de la persona para crear significado y comunicarse en la vida social. Según lo declarado por Otheguy et al (2015), el punto que necesita repetirse es que un idioma no puede definirse lingüísticamente, no puede definirse, es decir, en términos gramaticales (léxicos o estructurales). Debido a que un idioma no puede definirse lingüísticamente, no es, estrictamente hablando, un objeto lingüístico; no es algo de lo que una persona habla.

Por lo tanto, la comprensión de nuevas ideas sobre el uso del lenguaje es necesaria en la pedagogía del lenguaje y en las prácticas de enseñanza de idiomas, incluso cuando los estudiantes hablen el mismo idioma, ya que su uso, dependiendo de la situación, variará. Como consecuencia, un creciente campo de investigación en lingüística sociocultural impulsa la consideración de la interacción plurilingüe como una

1 Idioma es la lengua perfectamente desarrollada de un país en comunidad. Lenguaje es el sistema lingüístico de una comunicad. 
norma más que como una excepción (Gee y Handford, 2013). La discusión y el desafío son, entonces, mostrar cómo se puede expandir el lenguaje para retratarlo realmente como se usa en la sociedad, cuando se aprende en un entorno restringido como un aula.

Fairclough (2013), Palmer, Martínez, Mateus, \& Henderson (2014), entre otros, argumenta que el lenguaje debe ser visto, en la pedagogía de idiomas, como una actividad más que como una formación de palabras, más que simplemente un sistema al que recurrimos, como una parte ingeniosa de la vida social e intercultural en cualquier comunidad. Por lo tanto, los académicos antes mencionados, entre otros, argumentan que académicos y educadores de idiomas deberían alejarse de categorizaciones y conceptos fijos que apuntan a una perspectiva estática y unidimensional del lenguaje y, en cambio, intentar reemplazar estas ideas con términos que tengan más precisión para las prácticas lingüísticas en la vida cotidiana de las personas.

Haciéndose eco de las palabras de Canagarajah (2013), "la comunicación trasciende los idiomas individuales". Se puede argumentar, entonces, que se necesita un cambio con respecto a la enseñanza de idiomas, ya que algunas teorías, como las plurilingües, son la realidad hoy en día. El campo del lenguaje debe centrarse en la conjunción de los idiomas en la vida cotidiana de las personas. Comprender este último precepto puede ayudarnos a comenzar a pensar en nuevas formas de abordar la pedagogía de idiomas que ayuden a mejorar las capacidades lingüísticas de los estudiantes en las escuelas. Especialmente, cuando la enseñanza de un idioma extranjero es obligatoria dentro del currículo de escuelas públicas o privadas.

\section{DESARROLLO}

Dados los contextos globalizados en los que vive la mayoría de la población mundial (virtuales y físicos), y en respuesta a las nuevas necesidades educativas, diversos sistemas educativos alrededor del mundo han implementado, en algunos casos, programas de educación de enseñanza de idiomas. A veces, en reacción a la presión social y otras como parte del esfuerzo para promover la competitividad económica (Tallerman y Gibson, 2012). Esa es la razón por la cual muchos países de todo el mundo han participado en debates para determinar los idiomas en los que sus poblaciones deben ser educadas o deben aprender. En Nicaragua, por ejemplo, tradicionalmente la educación de un segundo idioma (ingles) ha sido parte de la educación de secundaria por décadas². Sin embargo, la enseñanza obligatoria de dicho idioma se ha establecido, paulatinamente, a partir de primaria en los últimos años.

En este mundo globalizados y en esta economía del conocimiento ${ }^{3}$, los idiomas juegan un papel ya que se entiende que aquellas personas exitosas son aquellas que poseen las habilidades para participar global y críticamente en diversos contextos. Actualmente, el país está en el centro de discusiones sobre la enseñanza del inglés en varios niveles de primaria ${ }^{4}$. Con la necesidad de dominio del inglés, ya sea para la movilidad social y/o profesional, surge la necesidad de una mejor implementación de modelos pedagógicos de enseñanza de idiomas extranjeras. El inglés en el aula parece tener más una función

2 https://www.mined.gob.ni/biblioteca/product-category/asignatura/english/

3 El economista y el teórico organizacional lo entienden como una economía construida sobre el trabajo del conocimiento. Es decir, la memorización de hechos y prácticas es insuficiente para el éxito socioeconómico (Sawyer, 2006)

4 www.mined.gob.ni/fortalecimiento-del-aprendizaje-de-ingles-en-educacion-primaria/ 
simbólica (por ejemplo, leer y escribir) y una función interpersonal (por ejemplo, conexión con diferentes grupos lingüísticos y culturales) (Alm, 2003; Paiva y Pagano, 2001). Fuera del aula, el lenguaje parece tener una función más innovadora (por ejemplo, arte, música). Comprender cómo se usan los idiomas según el contexto y el entorno es un elemento clave para comprender las prácticas lingüísticas, en cualquier contexto.

Comprender como se usan los idiomas funcionalmente implica, potencialmente, pensar en ideologías lingüísticas. Académicos han tratado las ideologías del lenguaje como un aspecto social, político e incluso lingüísticamente significativo. El concepto de ideología del lenguaje es escurridizo y desafía una definición clara en toda la academia. Varias definiciones del término se centran en diferentes aspectos del concepto, tales como valores o creencias con respecto al idioma de los hablantes en diferentes entornos (Johnson, 2016). Blackledge (2008) argumenta que las ideologías lingüísticas están unidas a las relaciones de poder y las influencias políticas. El autor ejemplifica su argumento al señalar que los idiomas de alto estatus ofrecen a sus hablantes capital político y cultural. Estos lenguajes de gran prestigio otorgan a todos los que desean aprender una moneda monetaria (real o simbólica) con ingresos socioeconómicos o socioculturales (Evans, 2015). Podemos argumentar, entonces, que las ideologías del lenguaje son creencias sociales sobre el estado del lenguaje en la sociedad y sobre cómo el idioma debe ser llevado o practicado por los miembros de su comunidad. Durante las últimas décadas, el concepto de ideología del lenguaje ha adquirido una fuerza considerable en diferentes áreas de la academia que tienen como objetivo desentrañar las idiosincrasias del lenguaje en el contexto de las prácticas sociales (Milani y Johnson, 2010).

Por lo tanto, cuando se trata del estudio del lenguaje y su relación con los comportamientos culturales, no podemos apartar las ideologías o el discurso sobre el uso del lenguaje en la sociedad. Me gustaría señalar que las ideologías y el discurso no se usan como sinónimos aquí. Como afirma Wood (2006), el discurso es la combinación de lenguaje y contexto. Cuando hablamos de contexto, nos referimos a las experiencias, expectativas y suposiciones que aportamos a la interacción con los demás. Dicha interacción se construye y negocia constantemente a través de las prácticas sociales en las que participamos. Es justo decir, entonces, que los discursos sociales son algo prescriptivos, por lo que alguien tiene la intención de decirle a otros cómo hablar, escribir o usar el idioma en ciertos contextos. La forma en que grupos de personas en comunidades específicas utilizan el lenguaje es importante para comprender cómo se construye el significado en función de las prácticas lingüísticas de las personas socialmente. Las ideologías derivan de las convenciones, creencias y valores del sistema que se dan por sentados y que se comparten entre sí por una comunidad. Como señala Gal (2006), las ideas y presupuestos culturales están etiquetados, y las personas usan esas etiquetas para evaluar diferentes grupos sociales y prácticas lingüísticas. El lenguaje no se usa en un vacío; más bien, se usa en contextos que están impregnados con la ideología de los sistemas sociales y políticos, y las instituciones sociales.

Como el lenguaje opera dentro de esta dimensión social, refleja ideologías construidas. Las ideologías del lenguaje incluyen las conceptualizaciones de las personas sobre la naturaleza de un idioma, como variedades y conexiones con entidades sociales notables, creencias sobre la superioridad o inferioridad de una variedad dada, creencias sobre la idoneidad de las mismas en una determinada situación o en relación con ciertos grupos de personas. En la educación de idiomas, particularmente, las ideologías lingüísticas 
representan intentos incompletos de racionalizar el uso del lenguaje. Dichas racionalizaciones suelen estar vinculadas al contexto y construidas a partir de las experiencias socioculturales de los hablantes, normalmente en una posición de poder (por ejemplo, responsables políticos, profesores) (Kroskrity, 2004; Duranti, 2008). Dichas ideologías se refieren tanto a la naturaleza del lenguaje como a la forma en que debe realizarse. El uso del término ideologías, en lugar de términos más neutrales como cultura, creencias o actitudes, apunta a una conformidad teórica sobre los puntos de vista de las personas sobre el lenguaje, que están moldeados por intereses políticos y económicos y, por lo tanto, el dominio y la subordinación (Philips. (1998) Como señala Jackson (2008), las ideologías lingüísticas dan forma y restringen el discurso y, por lo tanto, reproducen otros tipos de ideologías, como las ideologías de género, raza y clase. En otras palabras, se plantea una relación causal directa entre las ideas sobre el lenguaje y el uso real del lenguaje en la interacción. Las ideologías lingüísticas tradicionales restringen lo que las personas realmente hacen con el lenguaje (Philips y Oswick, 2012), pero también limitan cómo se enseñará un idioma y qué teoría pedagógica se utilizará para conducir al desempeño real del lenguaje en la sociedad (Fairclough, 2013).

Es importante tener en cuenta que el lenguaje se puede utilizar para transformar a los participantes; creencias y acciones en términos que son significativos para la institución que usa ese lenguaje. La educación es también, e indudablemente, el entorno institucional en el que las ideologías del lenguaje son más explícitas y más centradas en el lenguaje prescriptivo (Jackson, 2008); ya que sigue un enfoque normativo con respecto al uso del lenguaje en las escuelas. El lenguaje es una construcción ideológica que acomoda una considerable hibridación lingüística. Por lo tanto, las ideologías del lenguaje afectan las actitudes hacia los procesos lingüísticos, dialectos, registros y otras formas de prácticas lingüísticas. Adicionalmente, afectan valores, normas, estándares, lealtad del lenguaje, prestigio o estigmatización dentro de diferentes comunidades. El lenguaje no es simplemente un medio instrumental, las personas tienden a discutir sobre ideas, ideologías e identidades, no solo sobre la base de los canales en los que se expresan (Edwards, 2010). Comprender las ideologías del lenguaje sienta las bases para acceder y explorar las ideologías del lenguaje en un entorno de enseñanza de idiomas, que se caracteriza por cómo se utiliza el lenguaje según el contexto de las personas y con quién se comunica.

\section{CONCLUSIONES}

La forma en que se ve el lenguaje hoy es drásticamente diferente de la forma en que se percibió hace décadas. El concepto ha pasado de la idea de un sistema autónomo inamovible a un sistema adaptativo complejo con una función fundamentalmente social. En educación, el lenguaje juega un papel vital ya que el mundo se conecta constantemente a través de la tecnología, el comercio, la inmigración y la educación. El mundo globalizado requiere individuos que se esfuercen por crear espacios de conversación, negociación y mediación. Con un mundo tan plurilingüe en el que la gente vive hoy, han llegado nuevos desafíos para la educación, cómo educar a las personas en un mundo globalizado diverso.

En la pedagogía de idiomas, entender que el lenguaje es un sistema adaptivo complejo con una función fundamental social es vital. Implica entender cuál es la función que dicho idioma jugaría en diferentes aspectos de la sociedad. Lo que implica, consecuentemente, pensar que competencias deben ser desarrolladas dentro del aula de clase. Idealmente, los educadores de idiomas deben alejarse de conceptos fijos que apunten a una perspectiva estática y unidimensional del lenguaje y, en cambio, 
intentar reemplazar estas ideas con términos que tengan más precisión para las prácticas lingüísticas en la vida cotidiana de las personas. El uso funcional del lenguaje, su estudio y aprendizaje, conlleva abordar su relación con comportamientos culturales. Como el lenguaje opera dentro de esta dimensión social, refleja ideologías construidas. Las ideologías del lenguaje incluyen las perspectivas de las personas sobre la naturaleza de un idioma, por ejemplo, las creencias sobre la idoneidad de las mismas en una determinada situación o en relación con ciertos grupos de personas.

Indudablemente, el campo de la enseñanza se ha reubicado, en su enfoque, en las últimas décadas. Nos hemos movido del enfoque centrado en el maestro, en el que el papel del maestro era básicamente hablar o dar pláticas (transmitir conocimiento) mientras el estudiante recibía información de forma pasiva, a una experiencia de aprendizaje más dinámica. En estos días, las personas en educación buscan participar y construir significados enfocándose en cambios pedagógicos y experiencias más centradas en el alumno en lugar de una pedagogía tradicional de simplemente dar una charla. Con el idioma inglés influyendo profundamente en la educación en el país, la discusión de pedagogías más inclusivas para ayudar a los estudiantes se ha vuelto más relevante que nunca. En educación, especialmente, las pedagogías de idiomas, como el inglés, pueden ayudar mucho a los estudiantes al proporcionar herramientas para que sean más competitivos en un mundo globalizado en constante expansión. Por lo tanto, entender las nuevas visiones del 'lenguaje' es un paso importante para empezar a pensar en los cambios necesarios que se deben llevar a cabo en la pedagogía de idiomas.

\section{REFERENCIAS}

Alm, C. O. English in the Ecuadorian commercial context. World Englishes, Hoboken, v. 22, n. 2, p. 143-158, 2003.

Blackledge, A. Language ecology and language ideology. In: Stephen, M. (Ed.). Encyclopedia of language and education. Boston: Springer, 2008. p. 2923-2936.

Canagarajah, s. Translingual practice: global Englishes and cosmopolitan relations. London: Routledge, 2013.

Duranti, a. (Ed.). A companion to linguistic anthropology. Oxford: John Wiley \& Sons, 2008.

Edwards, J. Minority languages and group identity: cases and categories. Amsterdam: J. Benjamins Publishing, 2010.

Ellis, N. C.\& Larsen-Freeman, D. Language as a complex adaptive system. Hoboken: Wiley-Blackwell, 2009. Evans, D (Ed.). Language and identity: Discourse in the world. Bloomsbury: Publishing, 2016.

Fairclough, N. Critical discourse analysis: the critical study of language. Abingdon-on-Thames: Routledge, 2013.

Gal, s. Migration, minorities and multilingualism: Language ideologies in Europe. In Language ideologies, policies and practices, 2006, p. 13-27. Palgrave Macmillan, London.

Garcia, o. \& Wei, b. Translanguaging and education. In: Translanguaging: language, bilingualism and education. United Kingdom: Palgrave Macmillan, 2014. p. 63-77.

Gee, J. P. \& Handford, M. The Routledge handbook of discourse analysis. New York: Routledge, 2013.

Jackson, J. K. The social construction of youth and mathematics: the case of a fifth-grade classroom. In: Martin, D (Ed.). Mathematics teaching, learning, and liberation in the lives of Black children. New York: Routledge, 2008, pp. 185-209. 
Johnson, k. Language teacher education. In: HALL, G. (Ed.). The Routledge handbook of English language teaching. London: Routledge, 2016. p. 121-134.

Kroskrity, P. V. Language ideologies. In: DURANTI, A. (Ed.). A companion to linguistic anthropology. Malden: Blackwell Publishing, 2004. p. 496-517.

Migge, B.\& Léglise, I. Exploring language in a multilingual context: variation, interaction and ideology in language documentation. [S.I.]: Cambridge University Press, 2012.

Milani, T. M. \& Johnson, S. Critical intersections In: MILANI, T. M.; JOHNSON, S. Language ideologies and media discourse: texts, practices, politics. [S.I.]: Continuum, 2010, p. 3-14.

Otheguy, R., Garcia, O. \& Reid, W. Clarifying translanguaging and deconstructing named languages: a perspective from linguistics. Applied Linguistics Review, Berlin, v. 6, n. 3, p. 281-307, 2015.

Paiva, V. L. M. O. \& Pagano, A. S. English in Brazil with an outlook on its function as a language of science. In: AMMON, U. (Ed.). The dominance of English as a language of science: effects on other languages and language communities. Berlin: Mouton de Gruyter, 2001. p. 425-445.

Palmer, D. K. et al. Reframing the debate on language separation: toward a vision for translanguaging pedagogies in the dual language classroom. The Modern Language Journal, Oxford, v. 98, n. 3, p. 757-772, 2014.

Philips, S. U. Ideology in the language of judges: how judges practice law, politics, and courtroom control, v. 17. Oxford: Oxford University Press, 1998.

Phillips, N.\& Oswick, C. Organizational discourse: domains, debates, and directions. The Academy of Management Annals, v. 6, n. 1, p. 435-481, 2012.

Sawyer, R. K. (2006). Educating for innovation. Thinking skills and creativity, 1(1), 41-48.

Tallerman, M. \& Gibson, K. R. (Ed.). The Oxford handbook of language evolution. Oxford: Oxford University Press, 2012.

Wood, T. (2006). Adherence relations in literary and non-literary discourse.Journal of literary semantics, 35(2), 165-180. 